\title{
ORIGEN, DOMESTICACIÓN Y DIVERSIFICACIÓN DEL FRIJOL COMÚN. AVANCES Y PERSPECTIVAS
}

\section{ORIGIN, DOMESTICATION AND DIVERSIFICATION OF COMMON BEANS. ADVANCES AND PERSPECTIVES}

\author{
Víctor M. Hernández-López¹, Ma. Luisa P. Vargas-Vázquez², José S. Muruaga-Martínez², \\ Sanjuana Hernández-Delgado ${ }^{1}$ y Netzahualcóyotl Mayek-Pérez ${ }^{1 \star}$
}

\begin{abstract}
${ }^{1}$ Centro de Biotecnología Genómica, Instituto Politécnico Nacional. Blvd. del Maestro esq. Elías Piña s/n, Col. Narciso Mendoza, 88710, Reynosa, Tamaulipas. ${ }^{2}$ Campo Experimental Valle de México, Instituto Nacional de Investigaciones Forestales, Agrícolas y Pecuarias. 56230, Chapingo, México.
\end{abstract}

${ }^{*}$ Autor para correspondencia (nmayek@ipn.mx)

\section{RESUMEN}

Entre los años 9000 y 5000 a. C. en diferentes partes del mundo se domesticaron diversas especies vegetales, entre ellas el frijol común (Phaseolus vulgaris $\mathrm{L}$ ). La importancia de identificar el centro de origen y de domesticación de una especie como $P$. vulgaris radica en que esas áreas son fuente primaria de poblaciones con genes útiles para el mejoramiento genético y de interés para el entendimiento de la evolución, diversificación y conservación de la especie. El conjunto de conocimientos recabados hasta hoy, como la edad de los restos fósiles y las características morfológicas, agronómicas y genéticas, establecen que el frijol común se originó en Mesoamérica y posteriormente se domesticó entre los 5000 y 2000 años a. C. en dos sitios del continente Americano: Mesoamérica (México y Centroamérica) y los Andes (Sudamérica). A partir del frijol silvestre se formaron dos acervos genéticos domesticados distintos, Mesoamericano y Andino. El uso de nuevas herramientas biotecnológicas y genómicas han ofrecido evidencias definitivas sobre el origen, domesticación y diversidad de P. vulgaris.

Palabras clave: Phaseolus vulgaris, domesticación, acervos genéticos y razas, centros de origen y diversidad, recursos genéticos.

\section{SUMMARY}

Neolithic revolution took place from 9000 to 5000 years b. C., and at different sites in the world several plants species were domesticated, among them the common bean (Phaseolus vulgaris $\mathrm{L}$ ). Location of the origin and domestication centers of $P$. vulgaris is important to identify areas where major sources of populations carrying useful genes for plant breeding exist and because such populations could improve our understanding on the evolution, diversification and conservation of the species. The accumulated knowledge on fossil dates and other morphological, agronomic and genetic traits indicates that the common bean was originated at the Mesoamerican region, and afterwards it was domesticated from 5000 to 2000 years b. C. in two sites in the American continent: Mesoamerica (México and Central America) and the Andean region (South America). The use of new tools based on molecular technologies and genomics have given definitive evidences on the origin, domestication and genetic diversity of P. vulgaris.

Index words: Phaseolus vulgaris, domestication, gene pools and races, centers of origin and diversity, genetic resources.

\section{INTRODUCCIÓN}

Nuestros antepasados fueron cazadores-recolectores durante más de un millón de años. En el periodo de 3000 a 8000 años a. C. aparecieron las primeras sociedades humanas sedentarias capaces de producir sus alimentos y comenzaron a domesticar una gran variedad de plantas y animales en diferentes regiones del mundo, entre ellas Mesoamérica y los Andes en el continente Americano (Figura 1) (Smith, 2006; Zizumbo-Villarreal y Colunga-GarcíaMarín, 2010). La domesticación de plantas y animales contribuyó a " $\mathrm{La}$ Revolución Neolítica” que marcó un punto importante en la historia del hombre pues la agricultura se convirtió en la base de la economía.

La domesticación de plantas es un proceso resultante de la combinación de la evolución natural y la selección empírica practicada por el hombre, mediante la cual se derivan los cultivos domesticados a partir de sus progenitores silvestres. En general, se entiende que la domesticación de las plantas es un proceso dinámico y en curso (Zohary, 1999; Gepts, 2004), y es de sumo interés identificar los sitios originales de domesticación de un cultivo desde el punto de vista evolutivo, así como de importancia práctica para los fitomejoradores y conservacionistas.

Las domesticaciones múltiples en tiempo y espacio han sido determinantes claves en la estructura de la diversidad genética presente en los cultivos actuales. Ejemplos documentados de domesticaciones múltiples en distintas especies en el Continente Americano son el chile (Capsicum sp.) (Pickersgill, 1989), la calabaza (Cucurbita sp.) (Andres, 1990; Smith, 1997; Sanjur et al., 2002) y el frijol (Phaseolus sp.) (Gepts, 1988; Zizumbo-Villarreal y Colunga-GarcíaMarín, 2010).

En frijol la domesticación redujo la diversidad genética por un fenómeno denominado "cuello de botella de la domesticación”, que consiste en la reducción de la diversidad 


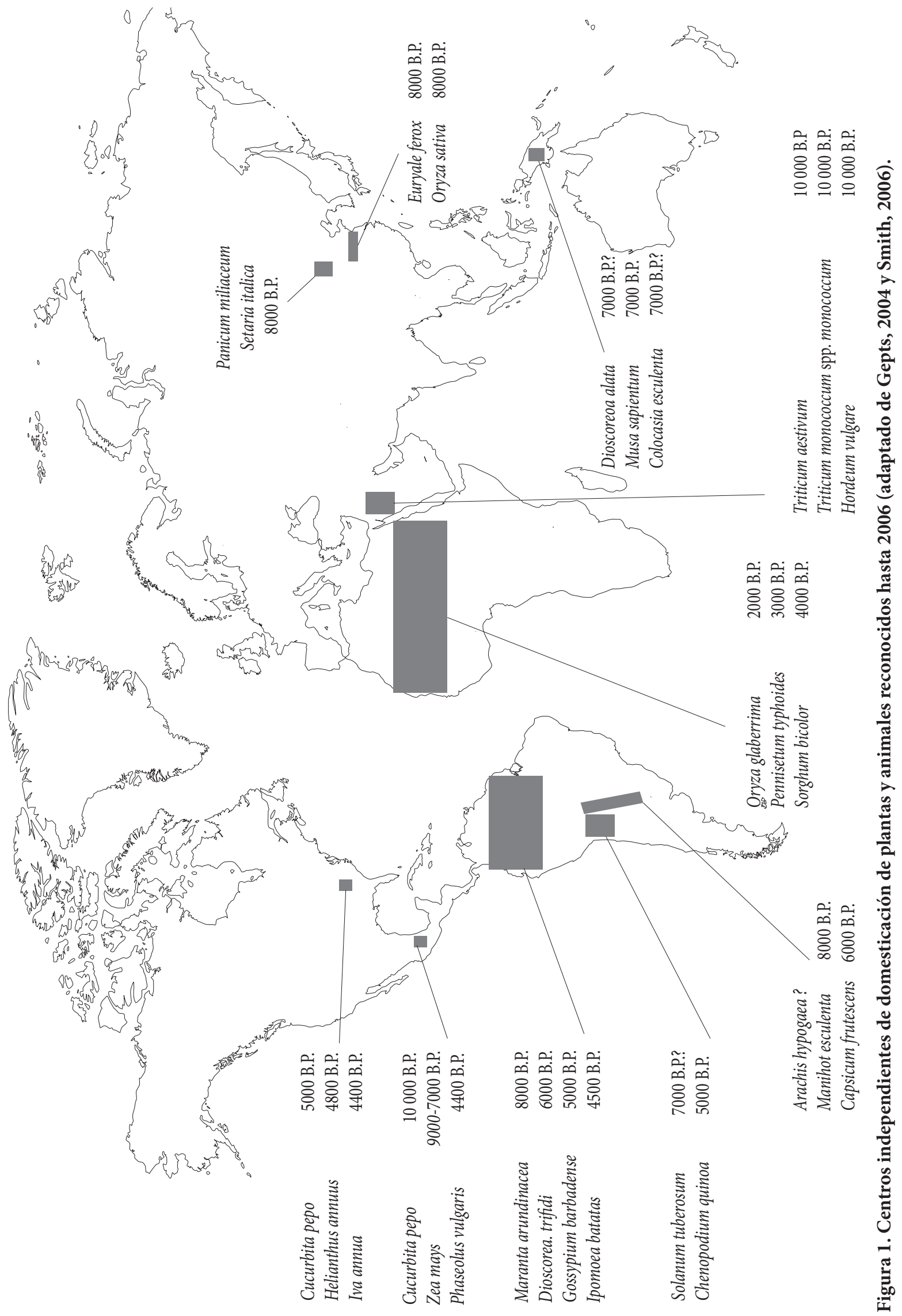


genética de la población en comparación con su ancestro, debido al pequeño número de individuos que fundaron las poblaciones domesticadas (efecto fundador) (Ladizinsky, 1998), y la posterior selección con base en características particulares que redujeron aún más la diversidad genética de loci específicos y regiones genómicas circundantes (Papa et al., 2006).

Para determinar el origen y la domesticación de las plantas suelen utilizarse cuatro tipos de evidencias, de acuerdo con de Candolle (1882) (citado por Gepts, 1999). Éstas son las arqueológicas (más específicamente arqueobotánicas); las botánicas (por ejemplo, la distribución de las especies silvestres o de sus parientes ancestrales); las históricas (la existencia de registros escritos que documentan la importancia o existencia del cultivo); y las lingüísticas (por ejemplo, la existencia de palabras que designen el cultivo o conceptos relacionados con el cultivo en lenguaje autóctono).

El género Phaseolus incluye cinco especies domesticadas: P. vulgaris (frijol común), P. lunatus (frijol lima), $P$. acutifolius (frijol tépari), $P$. coccineus ssp. coccineus (frijol ayocote) y $P$. dumosus $=P$. polyanthus $(=P$. coccineus $\mathrm{ssp}$. darwinianus) (frijol de año) (Freytag y Debouck, 2002). Los primeros trabajos sobre el origen y evolución del frijol se remontan a Miranda-Colín (1967) y Gentry (1969), quienes afirmaron que la forma silvestre de frijol se encuentra en Mesoamérica. Posteriormente, se han propuesto centros de origen y domesticación alternativos que trabajos sucesivos han ratificado o rectificado (Kwak et al., 2009).

El frijol común comprende dos acervos genéticos, el Mesoamericano y el Andino, que difieren en sus estructuras y niveles de diversidad genética, tanto en poblaciones silvestres como en las domesticadas. La mayor diversidad del germoplasma Mesoamericano se fundamenta en sus mayores proporciones del componente de varianza interpoblacional y diferenciación genética en el germoplasma domesticado (Beebe et al., 2000; Beebe et al., 2001; Papa y Gepts, 2003). En parte esto se debe a que poblaciones de germoplasma Mesoamericano de P. vulgaris son simpátricas con otras especies sexualmente compatibles como $P$. coccineus, lo que puede provocar hibridación interespecífica o introgresiva. Otros factores que afectan los niveles de diversidad son el aislamiento reproductivo parcial entre acervos genéticos, la homoplasia y la evolución convergente, aunque permanecen sin estudiarse a fondo (Papa et al., 2006).

En los últimos años se ha demostrado que la información generada mediante la aplicación de herramientas de marcadores moleculares de $\mathrm{ADN}$ ha incrementado el entendimiento sobre la diversidad genética y el proceso de domesticación del frijol común (Mensack et al., 2010). El reciente desarrollo de técnicas como los marcadores microsatélites y de secuencias de etiquetas sencillas en ADN cloroplástico y mitocondrial, así como la de secuenciación del genoma, ayudarán en la identificación de las rutas de migración del frijol en el mundo y permitirán distinguir el papel evolutivo de la selección, deriva y migración. Al respecto, se debe tener en cuenta que fuerzas como la migración y la deriva afectan loci distribuidos a través del genoma, mientras que la selección sólo afecta loci específicos debido a la recombinación (Papa et al., 2006; Bitocchi et al., 2013).

Un concepto adicional podría ser el análisis cariotípico del frijol, mediante el cual se ha constatado que el genoma de la especie evoluciona debido a la ocurrencia de inversiones peri y paracéntricas, traslocaciones y a la pérdida o ganancia de cromatina, así como por aneuploidía. Recientes avances indican que el cariotipo de $P$. vulgaris tiende a la asimetría y a la reducción del contenido de cromatina total, y que los altos contenidos de ADN se relacionan con mayor adaptación de la especie a regiones frías y templadas, mientras que los bajos contenidos de ADN se asocian con la adaptación a ambientes secos y cálidos (MercadoRuaro y Delgado-Salinas, 2000). Desafortunadamente, los estudios citogenéticos en frijol común no son consistentes y aún falta mucho que estudiar al respecto.

En el presente documento se revisan y discuten los estudios y trabajos que han contribuido a determinar el origen, la domesticación y la diversificación del frijol común, con énfasis en los relativos a arqueología, botánica sistemática, agronomía, bioquímica y biología molecular.

\section{ENFOQUE ARQUEOLÓGICO}

La arqueología es la ciencia que estudia los cambios producidos en las sociedades humanas a través de los restos materiales distribuidos en el espacio y contenidos en el tiempo. Dicho estudio se basa en el análisis arqueológico, subdividido a su vez en la prospección, la excavación y el análisis de gabinete. En laboratorio, una parte importante es la caracterización del material, su preservación y registro, así como su datación (Gamble, 2002). Una de las estrategias actuales y precisas para la datación de restos es el uso de técnicas como el ${ }^{14} \mathrm{C}$, cuyos resultados ayudan a entender el origen y domesticación de las plantas cultivadas. Los restos más antiguos de $P$. vulgaris se encontraron en el Continente Americano, en dos áreas geográficas lejanas: Mesoamérica y los Andes (Kaplan, 1965; Kaplan et al., 1973).

Mesoamérica fue centro de domesticación de cultivos importantes: maíz (Zea mays), frijol (P. vulgaris), calabaza (C. pepo), chile (Capsicum sp.) y caca o (Theobroma cacao) 
(Zizumbo-Villarreal y Colunga-GarciaMarín, 2010). Kaplan (1965) estableció que $P$. vulgaris se domesticó en el Valle de Tehuacán, Puebla, México hace aproximadamente 7000 años, probablemente en asociación con el maíz. En las cuevas ‘El Guitarreo’ y ‘El Callejón’ en Hualyas, Perú se recuperaron restos de $P$. vulgaris, con características similares a las formas actuales cultivadas de frijol. Esto indica que Perú pudo ser uno de los primeros centros de domesticación del frijol. El análisis de los restos de plantas con base en la prueba del ${ }^{14} \mathrm{C}$ indicó que la edad media del $P$. vulgaris domesticado es de $7680 \pm 280$ años (Kaplan et al., 1973).

El análisis de los vestigios de frijol, maíz y calabaza encontrados en cuevas de México (en los Estados de Tamaulipas,
Puebla y Oaxaca) por medio del método del radiocarbono y el uso de la espectrofotometría de masas con acelerador (AMS), reveló una edad de la forma domesticada del frijol más reciente y menor a 2400 años (Figura 2) (Smith, 2001; Smith, 2005).

La información recuperada de las cuevas permitió formular la hipótesis de que en las tierras altas ocurrió la domesticación de las plantas en Mesoamérica. La evidencia de que los primeros agricultores se asentaron en tierras bajas es más difícil de comprobar debido a que las condiciones climáticas, como humedad alta, no permiten la preservación de los restos. Pope et al. (2001) analizaron restos extraídos de excavaciones en San Andrés, Tabasco, entre ellas

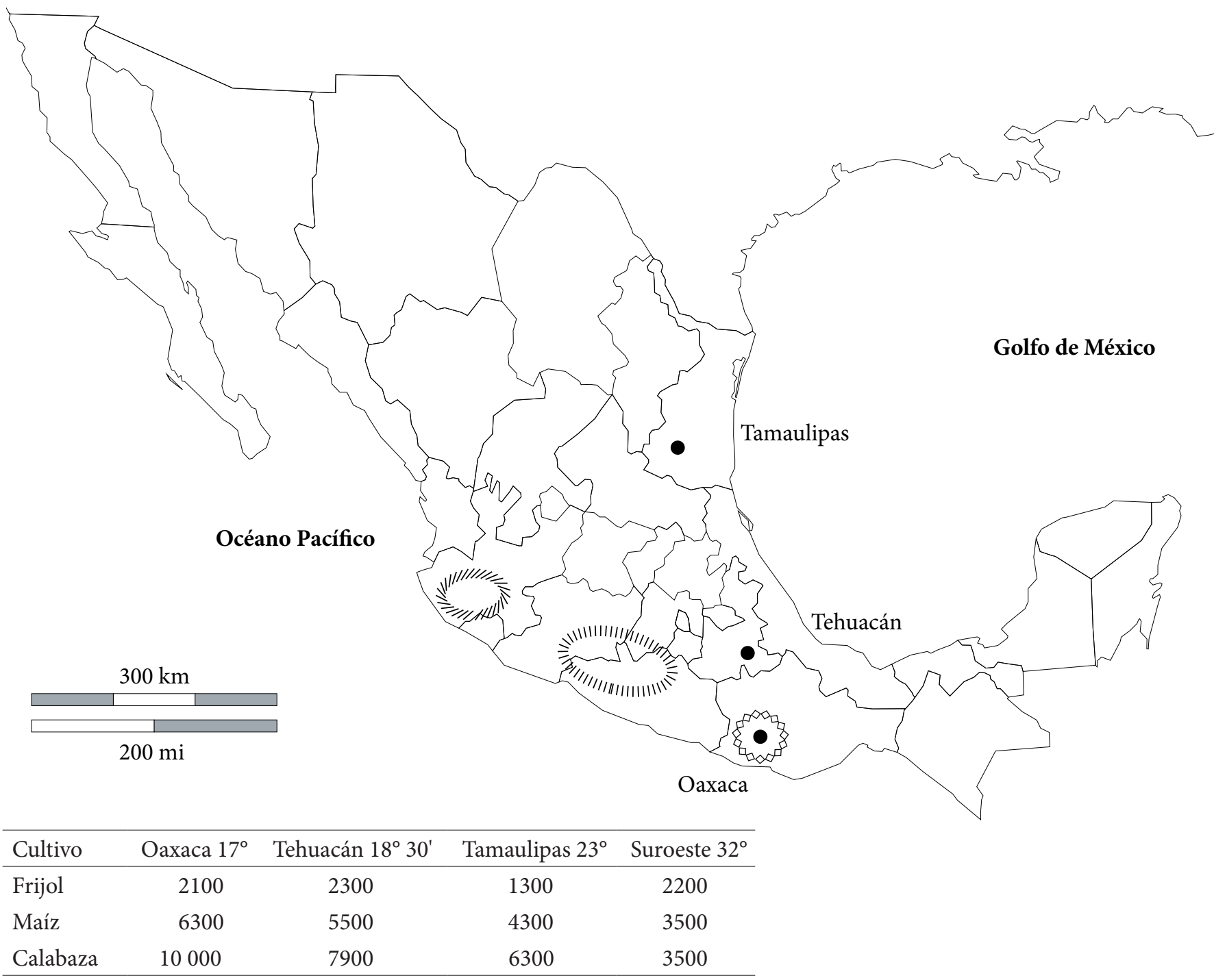

Figura 2. Mapa de México que muestra las áreas geográficas de domesticación de frijol común (////), maíz (| | | | | |) y calabaza $(\bowtie \diamond)$, así como la ubicación aproximada de las cuevas de Ocampo, Tamaulipas; Tehuacán, Puebla y Guilá Naquitz, Mitla, Oaxaca (Adaptado de Smith, 2001; Smith, 2005; Kwak et al., 2009). El cuadro inferior indica las latitudes de los sitios y las fechas de domesticación del frijol, maíz y calabaza. 
una semilla de Phaseolus sp. con edad de $2345 \pm 50$ años a. C., lo cual indica que la presencia de las primeras plantas domesticadas no solamente ocurrió en regiones altas sino que también en zonas de baja altitud, y que también influyeron en la transición de la forma de vida de las primeras sociedades humanas en Mesoamérica. En resumen, las evidencias y estudios arqueológicos soportan el origen Mesoamericano del frijol.

\section{ENFOQUES MORFOLÓGICO-AGRONÓMICO Y BIOQUÍMICO}

El género Phaseolus incluye especies leguminosas con alta importancia en el mundo desde el punto de vista alimenticio, que se desarrollan en una amplia variedad de condiciones ambientales: desde los $52^{\circ} \mathrm{LN}$ hasta los $32^{\circ}$ de LS, en los trópicos húmedo y semiárido, e incluso en regiones con clima frío (Schoonhoven y Voysest, 1991). El análisis de rasgos morfológicos y agronómicos en accesiones domesticadas provenientes de México, Guatemala, Nicaragua, El Salvador, Honduras, Costa Rica, República Dominicana, Colombia, Ecuador, Perú, Chile, Bolivia, Brasil y Argentina, explicaron los patrones de diversidad y las relaciones genéticas en ese germoplasma y permitieron diferenciar los acervos genéticos (Singh et al., 1991a).
Las características evaluadas en las accesiones de frijol domesticado han sido: rasgos de la semilla, rasgos de la etapa vegetativa, del desarrollo floral, fructificación, madurez fisiológica y resistencia a enfermedades (Singh et al., 1991a; Vargas et al., 2006). Las poblaciones domesticadas muestran el "síndrome de la domesticación", que se distingue por pérdida de la capacidad de dispersión y de la latencia, arquitectura compacta de la planta, mayor rendimiento de grano y semilla de mayor tamaño, sincronía y precocidad de la floración. Estos cambios son debidos a que los alelos relacionados con el "síndrome de la domesticación" muestran pérdida de función génica (Papa et al., 2006). Los resultados también indican la existencia de dos acervos genéticos en el germoplasma domesticado: el Mesoamericano y el Andino (Cuadro 1), aunque algunas accesiones del norte Andino muestran características distintas a los dos grupos genéticos antes mencionados (Singh et al., 1991a; Singh et al., 1991b; Islam et al., 2002a).

Los marcadores bioquímicos proveen información en tiempos más cortos y de características que no son tan influenciadas por el ambiente (Gepts, 1999). Algunas técnicas bioquímicas utilizadas en el frijol común se han dirigido a la determinación del tipo de faseolinas, lecitinas, inhibidores de $\alpha$-amilasa, aloenzimas o isoenzimas. El germoplasma silvestre es más diverso que el domesticado en cuanto

Cuadro 1. Diferencias en las características morfológicas y agronómicas de dos centros de origen y domesticación del frijol común.

\begin{tabular}{lcc}
\hline \multirow{2}{*}{ Característica } & \multicolumn{2}{c}{ Centro de origen y domesticación } \\
\cline { 2 - 3 } Forma de la hoja & Mesoamericano & Sur Andino \\
Pubescencia en las hojas & Acorazonada y ovalada & Lanceolada y aguda \\
Longitud del quinto internudo & Escasa y corta & Densa y larga \\
Inflorescencia & Corto & Largo \\
Forma de la bráctea & Varios nudos & Un solo nudo \\
Base del pétalo & Acorazonada y ovalada & Lanceolada y triangular \\
Posición del ápice de la vaina & Rayada & Lisa \\
Tamaño de la semilla & Placentaria & Central \\
Faseolina (\%) & Pequeña y mediana & Grande \\
Lecitinas (\%) & $037.8 \pm 5.90$ & $041.8 \pm 6.60$ \\
Calcio (\%) & $011.8 \pm 3.00$ & $010.5 \pm 2.20$ \\
Fosforo (\%) & $0.157 \pm 0.04$ & $0.144 \pm 0.04$ \\
Azufre (\%) & $0.376 \pm 0.06$ & $0.344 \pm 0.07$ \\
Fierro (\%) & $0.216 \pm 0.03$ & $0.208 \pm 0.02$ \\
Zinc (\%) & $053.4 \pm 7.80$ & $055.6 \pm 7.90$ \\
Adaptado de: Singh et al. (1991a), Singh et al. $(1991 b)$, Islam et al. (2002a). Los valores indican la media \pm la \\
desviación estándar.
\end{tabular}


a caracteres físico-químicos del grano. Un cambio importante que ocurrió con la domesticación fue el incremento sustantivo del tamaño del grano (gigantismo), la reducción de la dureza y del tiempo de cocción del grano, además de una marcada reducción en los contenidos de triptófano y proteínas. En cambio, la semilla del germoplasma silvestre es más rica en los contenidos de minerales y fibra dietética que el germoplasma domesticado (Acosta-Gallegos et al., 2007; Peña-Valdivia et al., 2011).

Como se indicó antes, los rasgos morfológicos han permitidodiferenciartresáreas principales de domesticación de P. vulgaris: Mesoamérica y Los Andes (Singh et al., 1991a; Singh et al., 1991b; Islam et al., 2002a). Las características morfológicas son contrastantes entre grupos y son útiles para diferenciarlos (Cuadro 1). El acervo Mesoamericano presenta menor concentración de faseolinas y lecitinas, y mayor contenido de elementos nutritivos con excepción del $\mathrm{Fe}$ (Cuadro 2). El germoplasma del Norte Andino muestran rasgos intermedios entre ambos grupos genéticos, aunque en algunos casos no sucede así (Islam et al., 2002a; Islam et al., 2002b).

La información de aloenzimas, faseolinas, lecitinas e inhibidores de $\alpha$-amilasa permite distinguir entre acervos Andino y Mesoamericano, y también la variabilidad adicional del Norte Andino (Koenig y Gepts, 1989; Islam et al., 2002b). El acervo Mesoamericano muestra mayor diversidad tanto de aloenzimas como de ciertos tipos de faseolinas. En contraparte, el Andino muestra diversidad genética reducida (Koenig y Gepts, 1989; Singh et al., 1991a; Singh et al., 1991b; Islam et al., 2002a; Islam et al., 2002b; Chacón et al., 2005). En conclusión, las evidencias morfo-agronómicas y bioquímicas muestran el origen Mesoamericano del frijol común así como la presencia de tres centros de domesticación: Mesoamericano, Norte Andino y Sur Andino.

\section{ENFOQUE MOLECULAR}

El análisis de las tasas evolutivas indica que el clado Phaseolus tiene una edad máxima de 6 a 8 millones de años y cada clado del género va de 1 ( $P$. filiformis, $P$. pedicellatus, $P$. polystachios; éstas dos últimas especies son las más ricas genéticamente en el género) a 4 millones de años (grupo Vulgaris) (Delgado-Salinas et al., 2006). La filogenia de Phaseolus se ha caracterizado con base en el análisis de marcadores "Internal Transcribed Spacer" (ITS) o "Espaciadores Internos Transcritos", morfológicos y citogenéticos. En este sentido, Phaseolus ha mostrado ser un género monofilético con dos linajes, uno que incluye las especies domesticadas $P$. vulgaris, $P$. coccineus, $P$. dumosus y $P$. acutifolius, así como $P$. albescens y $P$. costaricensis; el otro incluye a $P$. lunatus y especies silvestres de Mesoamérica y los Andes (Delgado-Salinas et al., 1991).

Posteriormente, Delgado-Salinas et al. (2006) ampliaron su estudio e incluyeron 52 especies de Phaseolus que analizaron con base en marcadores ITS de ribosomas nucleares y de un locus plastídico, trnK. Este estudio ratificó la formación de dos linajes o clados en Phaseolus. Uno incluye los grupos Tuckerheimii, Pauciflorus y Pedicellatus, y el otro a los grupos Filiformis, Vulgaris, Leptostachyus, Lunatus y Polystachios. Además, estos autores identificaron cinco especies 'enigmáticas': P. glabellus, P. macrolepis, P. microcarpus, $P$. oaxacanus y $P$. talamancesis, cercanas a Tuckerheimii.

La diversidad genética de los frijoles silvestre y cultivado se ha estudiado mediante marcadores moleculares de ADN, como el ADN polimórfico amplificado al azar (RAPDs), polimorfismos en la longitud de los fragmentos amplificados (AFLPs), secuencias de ADN, microsatélites o secuencias simples repetidas (SSRs) o polimorfismos en la longitud de los fragmentos de restricción (RFLPs) (Mensack et al., 2010). Todos o al menos la mayoría de estos marcadores

Cuadro 2. Comparación de marcadores bioquímicos y moleculares mostrados por los acervos genéticos de $P$. vulgaris.

\begin{tabular}{|c|c|c|}
\hline \multirow{2}{*}{ Característica } & \multicolumn{2}{|c|}{ Centro de origen y domesticación } \\
\hline & Mesoamericano & Andino \\
\hline Faseolina $^{\dagger}$ & $\mathrm{B}, \mathrm{M}, \mathrm{S}, \mathrm{Sb}, \mathrm{Sd}$ & $\mathrm{C}, \mathrm{Ca}, \mathrm{CH}, \mathrm{H}, \mathrm{Ko}, \mathrm{Pa}, \mathrm{T}, \mathrm{To}$ \\
\hline Lectinas & M, Po & $\mathrm{K}, \mathrm{T}, \mathrm{V}, \operatorname{Pr}, 312$ \\
\hline Inhibidor de $\alpha$-amilasa & A3, A7 & A1 \\
\hline Aloenzima & Lap- $3^{100}, \mathrm{Me}^{100}, \mathrm{Rbcs}^{100}$, Skdh $10^{103}$ & Lap-3 ${ }^{103}, \mathrm{Me}^{98}, \mathrm{Rbcs}^{98}$, Skdh $10^{100}$ \\
\hline ADN cloroplástico & $\mathrm{B}, \mathrm{H}, \mathrm{J}, \mathrm{K}, \mathrm{N}, \mathrm{O}$ & $C, D, E, F$ \\
\hline
\end{tabular}


pueden diferenciar claramente al germoplasma de $P$. vulgaris en dos centros de origen y domesticación (Cuadro 2) (Debouck, 1986; Koenig y Gepts, 1989; Singh et al., 1991a, 1991b; Sonnante et al., 1994; Islam et al., 2002a, 2002b; Chacón et al., 2005; Rossi et al., 2009); algunos otros estudios con marcadores moleculares sugieren un tercer centro de origen y domesticación de la especie, denominado el Norte Andino (Islam et al., 2002b).

El origen del frijol común es Mesoamericano y a partir del "cuello de botella de la domesticación", concepto descrito anteriormente, se formó el acervo Andino antes de la domesticación, que por migración se llevó a Sudamérica (Bitocchi et al., 2012). La discrepancia entre un origen o dos o más se debe a que en unos trabajos previos los investigadores analizaron una faseolina tipo I y supusieron que las filogenias de especies y de genes son idénticas. En el frijol esta relación filogenética no se cumple debido a que la distribución actual de la faseolina podría no reflejar su distribución ancestral, o bien a que la faseolina tipo I podría haberse extinguido en Mesoamérica o estar aún presente pero no en el germoplasma analizado a la fecha. Además, en la actualidad se conoce la compleja estructuración genética y geográfica del germoplasma Mesoamericano de frijol distribuido en el centro de México a través del eje neovolcánico transversal, cuna de la diversidad genética de $P$. vulgaris (Bitocchi et al., 2012).

La diversidad genética se redujo con la domesticación y esta reducción fue mayor en el germoplasma Mesoamericano que en el Andino, como resultado del "cuello de botella" ocurrido antes de la domesticación en los Andes (Rossi et al., 2009; Bitocchi et al., 2012). El análisis de germoplasma silvestre y domesticado del acervo Mesoamericano indica un solo evento de domesticación asociado con la región de la cuenca de los ríos Lerma-Santiago en Jalisco, México (Kwak et al., 2009; Mamidi et al., 2011), y otro distinto de la cuenca del Río Balsas donde se ubicó el centro de domesticación del maíz (Matsuoka et al., 2002).

Según Kwak et al. (2009), aunque los maíces y frijoles silvestres crecían juntos la domesticación ocurrió en sitios distintos para cada especie, y fue tiempo después que se reunieron en un solo sistema de cultivo. Se ha estimado que la población "cuello de botella efectiva" fue de aproximadamente $50 \%$ de la población base en cada acervo genético, y que dicho "cuello" ocurrió entre los 8200 y 8500 años y entre los 6300 a 7000 años en los acervos Mesoamericano y Andino, respectivamente; de ahí que el mayor desequilibrio de ligamiento sea en el primer acervo (Mamidi et al., 2011). Estos resultados sugieren también que las futuras investigaciones deben re-enfocarse al germoplasma del centro-occidente de México.
Contrario al reporte de Kwak et al. (2009), los de Bitocchi et al. $(2012 ; 2013)$ proponen que la domesticación del frijol común en el acervo Mesoamericano no ocurrió en el occidente de México sino en el Valle de Oaxaca, y que el del Acervo Andino fue entre el sur de Bolivia y el norte de Argentina en Sudamérica. Estas propuestas difieren también con la de Chacón et al. (2005) quienes sugirieron como centro de domesticación en Sudamérica a Perú, y con la de Beebe et al. (2001) quienes propusieron al norte de Argentina y el este de Bolivia. Los resultados de Bitocchi et al. $(2012 ; 2013)$ coinciden con lo sugerido por Zizumbo-Villarreal y Colunga-GarcíaMarín (2010), en el sentido de que el área primaria de domesticación del frijol es similar a la del maíz, y que ambas especies ya domesticadas se movieron con la migración humana a lo largo de los ríos de México.

El análisis conjunto transcriptómico, proteómico y metabolómico de $P$. vulgaris pudo diferenciar claramente al germoplasma con base en sus centros de domesticación; incluso, el análisis metabolómico pudo generar huellas genéticas únicas en el germoplasma analizado, de modo que las modernas estrategias "ómicas" se vislumbran como herramientas baratas y fácilmente escalables para analizar e identificar germoplasma por alto valor agronómico o nutricio, así como para la caracterización de la diversidad genética (Mensack et al., 2010).

Resultados recientes apuntan a ratificar el origen Mesoamericano y Sudamericano de $P$. vulgaris, que dieron lugar a los acervos genéticos actualmente conocidos. La domesticación ocurrió en forma independiente en cada región a partir del acervo local.

\section{DISCUSIÓN}

La transición de la caza y la recolección como forma de vida a la economía basada en la producción de alimentos vía la agricultura, tanto en Mesoamérica como en los Andes, fue un proceso de desarrollo largo y complejo (ZizumboVillarreal y Colunga-GarcíaMarín, 2010), que involucró la adopción y domesticación de diferentes plantas en regiones con ambientes diversos para solucionar los problemas de alimentación y adaptación al ambiente local (Smith, 2006). La determinación exacta de la edad de los restos de plantas cultivadas que aparecieron por primera vez en los registros arqueológicos en áreas y regiones diferentes del mundo, representan una base esencial para el entendimiento de esta transición (Smith, 2005).

El análisis de muestras de las primeras plantas domesticadas con el método convencional de análisis de radiocarbono de restos vegetales de las cuevas de Oaxaca, Puebla y Tamaulipas en México, y de Hualyas en Perú, indican 
que la domesticación de $P$. vulgaris ocurrió alrededor de 5000 años a. C. (Kaplan, 1965; Kaplan et al., 1973; Smith, 2001). El nuevo análisis con AMS indica que, en realidad, la domesticación fue más reciente en Mesoamérica (Smith, 2005).

Las investigaciones realizadas coinciden en identificar el origen del frijol en Mesoamérica, concretamente en México, desde Jalisco hasta Oaxaca, y de ahí la especie migró a Sudamérica. El aislamiento geográfico dio lugar a dos acervos genéticos: el Andino y el Mesoamericano. Los estudios con enfoques arqueológicos, morfológico-agronómicos, bioquímicos y moleculares ofrecen evidencias diferentes y, aparentemente, inconsistentes (Kaplan, 1965; Kaplan et al., 1973; Smith, 2001). Esto se atribuye a que no se han hallado nuevos restos arqueológicos que estudiar, en ocasiones debido al estudio de colecciones incompletas de germoplasma o a una estrategia incorrecta de estudio; por ejemplo, en el caso de los marcadores moleculares de ADN es importante considerar la tasa de mutación en los cálculos de los años transcurridos (Bitocchi et al., 2012).

En las últimas décadas los estudios arqueológicos fijaron su atención en Mesoamérica debido a su amplia riqueza cultural y a la importancia del frijol en la dieta de los habitantes de la región (Smith, 1997; Avendaño-Arrazate et al., 2004; Smith, 2005). Los análisis de los restos de plantas de las cuevas de Oaxaca, Puebla y Tamaulipas han permitido postular al frijol común como la última de las tres plantas de mayor importancia (maíz, calabaza, frijol) en ser domesticadas en Mesoamérica (Smith, 2005), y que ello tuvo un efecto aditivo en el tiempo y espacio sobre el maíz y la calabaza, pues contribuyó sustancialmente y de manera positiva en la dieta y la economía agrícola regional (Smith, 2001).

Los nuevos métodos de determinación de la edad de restos de plantas indican tiempos de domesticación menores que los del radiocarbono convencional. Las diferencias entre métodos abre el debate respecto a la calendarización, contexto y causas que originaron la domesticación de $P$. vulgaris en Mesoamérica y los Andes, y remarcan la necesidad de más excavaciones en las cuevas de los primeros agricultores y los valles de ríos donde se puedan encontrar fósiles de plantas (Smith, 1997). En el contexto arqueológico es limitada la cantidad de información actualmente disponible para explicar el inicio de la domesticación de plantas y por consiguiente el origen de la agricultura.

Con base en el análisis de rasgos fenotípicos también se han discernido dos áreas geográficas de domesticación de P. vulgaris: Mesoamérica y Los Andes (Singh et al., 1991a; Singh et al., 1991b; Islam et al., 2002a). El grano del acervo Mesoamericano presenta menor concentración de faseolinas y lecitinas, pero mayor contenido de elementos nu- tritivos con excepción del Fe. En cambio, el germoplasma del Norte Andino muestra rasgos intermedios entre ambos acervos genéticos (Islam et al., 2002a; Islam et al., 2002b). Dado que las accesiones criollas de los Andes por lo general muestran semillas grandes, podría suponerse que la baja concentración de los nutrimentos se debe a la dilución dentro del almidón al momento del llenado del grano (Islam et al., 2002a). Es decir, los resultados permiten sugerir que el Norte Andino corresponde a una zona de transición y de mezcla genética entre los acervos Andino y Mesoamericano.

Mediante el uso de los marcadores bioquímicos y moleculares también se han distinguido dos grupos genéticos para $P$. vulgaris, al igual que con el enfoque morfológicoagronómico (Rossi et al., 2009); y también sugieren al Norte Andino como un tercer centro de domesticación (Koenig y Gepts, 1989; Singh et al., 1991a; Singh et al., 1991b; Islam et al., 2002a; Islam et al., 2002b; Chacón et al., 2005). Los datos de aloenzimas, faseolinas, lecitinas e inhibidores de a-amilasa, diferencian el acervo Andino del Mesoamericano, y detectan variabilidad adicional en el Norte Andino que no se encuentra en los dos sitios anteriores (Koenig y Gepts, 1989; Islam et al., 2002b).

El acervo Mesoamericano muestra mayor diversidad de aloenzimas y de ciertos tipos de faseolinas. En contraparte, el acervo Andino muestra baja diversidad genética (Koenig y Gepts, 1989; Singh et al., 1991a; Singh et al., 1991b; Islam et al., 2002a; Islam et al., 2002b; Chacón et al., 2005). La diferencia entre estos dos acervos se atribuye a la autopolinización en frijol, que limita el movimiento de genes entre poblaciones y que se traduce en el incremento de la diferenciación genética entre acervos genéticos, así como al ya descrito efecto de "cuello de botella" de la domesticación.

Otro factor importante en la diferenciación es el aislamiento geográfico que propicia la adaptación a condiciones ambientales específicas (Koenig y Gepts, 1989). Algunas accesiones del Norte Andino muestran una base genética distinta a los dos acervos ya establecidos, pues por ejemplo presenta los tipos de faseolinas CH, S, T, L; lecitinas 312, Pr, B y K; e inhibidores de a-amilasa (A4, A5 y A6) (Islam et al., 2002a; Islam et al., 2002b). El tipo de faseolina $\mathrm{CH}$ del norte Andino es distintivo de las accesiones de Colombia.

Las poblaciones silvestres del norte Andino muestran alelos únicos que están ausentes en los dos acervos genéticos principales (Gepts y Bliss, 1985), como la faseolina $\mathrm{CH}$ en accesiones silvestres y cultivadas de Colombia. La propuesta de un centro en el Norte Andino se refuerza al detectarse la presencia de lectinas e inhibidores de $\alpha$-amilasa atípicos de los dos principales acervos genéticos, junto con rasgos morfológicos característicos entre los grupos de accesiones 
estudiadas (Islam et al., 2002a; Islam et al., 2002b). El Norte Andino presenta las faseolinas tipos $\mathrm{CH}$ y $\mathrm{C}$ en las accesiones silvestres, pero solo la $\mathrm{CH}$ en las domesticadas y en baja frecuencia, lo que indica restricciones en el proceso de domesticación el que a su vez dio origen a niveles limitados de diversidad genética.

El Norte Andino comprende el noreste de Colombia, al Ecuador y al norte de Perú (Koenig, y Gepts, 1989; Singh et al., 1991a; Singh et al., 1991b; Islam et al., 2002a; Islam et al., 2002b). Las accesiones domesticadas provenientes del Norte Andino reflejan la introgresión de ambos acervos genéticos a partir de los cuales se hizo la domesticación local. La introgresión del material genético de los acervos genéticos principales provocó que la integridad del germoplasma del Norte Andino se haya reducido drásticamente, de modo que solo permite considerar a esta región como una zona de transición (Islam et al., 2002a; Islam et al., 2002b).

El análisis evolutivo de frijol mediante herramientas moleculares (Rossi et al., 2009; Kwak y Gepts, 2009; Kwak et al., 2009; Mensack et al., 2010; Nanni et al., 2011; Mamidi et al., 2011; Bitocchi et al., 2012; 2013) enfatizan el origen Mesoamericano de $P$. vulgaris, y que de ahí se extendió posteriormente al resto del Continente Americano, para luego ser domesticado tanto en Mesoamérica como en Sudamérica. Esto se contrapone a las evidencias bioquímicas anteriormente expuestas, particularmente las referentes a los tipos de faseolina que son determinantes de tres regiones geográficas distintas de origen. Es decir, la distribución actual de la faseolina no refleja su distribución ancestral, ya sea porque la faseolina tipo I se extinguió en Mesoamérica o porque solamente sigue presente en germoplasma no analizado a la fecha. Otra evidencia indica que el locus de la faseolina está ligado al gen del tamaño de la semilla, por lo que la distribución de la faseolina en el germoplasma domesticado pudo haber sido afectada por la selección artificial durante la domesticación (Kwak y Gepts, 2009).

De manera global se podría indicar que a través de los años los resultados y conclusiones relativos al tópico que se ha revisado, analizado y discutido en este trabajo, han sufrido modificaciones y adecuaciones, particularmente porque en algunos casos el espectro de germoplasma que se analiza no es lo más amplio y representativo posible, las técnicas de análisis se han perfeccionado y, por ende, ahora son más precisas en la identificación, asociación o clasificación del germoplasma de Phaseolus estudiado. Todavía ChacónSánchez (2009) se refería al proceso evolutivo y de domesticación del frijol en los términos y con los conocimientos hasta ese momento publicados, que aquí hemos revisado. Como también lo hemos visto, los trabajos posteriores a ese año han "identificado" regiones alternativas de domesticación, pero los resultados varían en función del sistema de análisis o del germoplasma analizado en cada caso. Paulatinamente el mejoramiento o refinamiento de las nuevas técnicas de análisis de germoplasma, particularmente las genómicas y otras "ómicas" presentes y futuras, serán herramientas útiles en el estudio de la genética y genómica de la diversidad y la diversificación del género Phaseolus.

\section{CONCLUSIONES}

Las evidencias aportadas por estudios con enfoques arqueológico, morfológico-agronómico, bioquímico y molecular, indican que el frijol tiene su centro de origen en la región de Mesoamérica, particularmente en el occidente y sur de México (desde Jalisco hasta Oaxaca), y que hubo dos centros de domesticación: uno primario (Mesoamérica) y otro secundario (Sur Andino). Las evidencias actuales no permiten discernir claramente al Norte Andino como un centro de domesticación adicional.

\section{AGRADECIMIENTOS}

Al CONACYT, al programa PIFI del IPN y a Universia Santander-ECOES, por las becas para los estudios de Maestría en Ciencias en Biotecnología Genómica en el CBG-IPN del primer autor. Al FOMIX-Gobierno del Estado de Veracruz (clave 94070), al Fondo de Ciencia Básica del CONACYT (proyecto no. 181756) y al Instituto Politécnico Nacional (SIP 20080666), por el financiamiento otorgado para el desarrollo del proyecto.

\section{BIBLIOGRAFÍA}

Acosta-Gallegos J A, J D Kelly, P Gepts (2007) Prebreeding in common bean and use of genetic diversity from wild germplasm. Crop Sci. 47:S44-S59.

Andres T (1990) Biosystematics, theories on the origin, and breeding potential of Cucurbita ficifolia. In: Biology and Utilization of the Cucurbitaceae. D M Bates DM, R W Robinson, C Jeffrey (eds). Cornell University, New York. pp:102-119.

Avendaño-Arrazate C H, P Ramírez-Vallejo, F Castillo-González, J L Chávez-Servia, G Rincón-Enrique (2004) Diversidad isoen zimática en poblaciones nativas de frijol negro. Rev. Fitotec. Mex. 27:31-40.

Beebe S D, J Rengifo, E Gaitan, M C Duque, J Tohme (2001) Diversity and origin of Andean landraces of common bean. Crop Sci. 41:854-862.

Beebe S D, P W Skroch, J Tohme, M C Duque, F Pedraza, J Nienhuis (2000) Structure of genetic diversity between common bean land races of Middle America origin based on correspondence analysis of RAPD. Crop Sci. 49:264-273.

Bitocchi E, E Bellucci, A Giardini, D Rau, M Rodriguez, E Biagetti, R Santilocchi, P S Zeuli, T Gioia, G Logozzo, G Attene, L Nanni, R Papa (2013) Molecular analysis of the parallel domestication of the common bean (Phaseolus vulgaris) in Mesoamerica and the Andes. New Phytol. 197:300-313.

Bitocchi E, L Nanni, E Bellucci, M Rossi, A Giardini, P Spagnoletti Zeuli, G Logozzo, J Stougaard, P McClean, G Attene, R Papa (2012) Mesoamerican origin of the common bean (Phaseolus vulgaris L.) is revealed by sequence data. Proc. Nat. Acad. Sci. USA 109: E788-E796.

Chacón M I, S B Pickersgill, D G Debouck (2005) Domestication patterns in common bean (Phaseolus vulgaris L.) and the origin of 
the Mesoamerican and Andean cultivated races. Theor. Appl. Genet. 110:432-444.

Chacón-Sáncez M I (2009) Darwin y la domesticación de plantas en las Américas: el caso del maíz y el fríjol. Acta Biol. Colomb. 14:351-364.

Debouck D G (1986) Primary diversification of Phaseolus in the Americas: three centres?. Plant Genet. Res. Newslet. 67:2-6.

Delgado-Salinas A, R Bibler, M Lavin (2006) Phylogeny of the genus Phaseolus (Leguminosae): a recent diversification in an ancient landscape. Syst. Bot. 31:779-791.

Delgado-Salinas A, A T Turley, A Richman, M Lavin (1991) Phylogenetic analysis of the cultivated and wild species of Phaseolus (Fabaceae). Syst. Bot. 24:438-460.

Freytag G F, D G Debouck (2002) Taxonomy, distribution, and ecology of the genus Phaseolus (Leguminosae-Papilionideae) in NorthAmerica, Mexico and Central America. SIDA, Botanical Miscellany 23. Botanical Research Institute of Texas. Fort Worth, USA. 300 p.

Gamble C (2002) Arqueología Básica. Ariel. Barcelona. 246 p.

Gentry H S (1969) Origin of the common bean, Phaseolus vulgaris. Econ. Bot. 23:55-69.

Gepts P (1988) Genetic resources of Phaseolus beans: Current plant science and biotechnology in agriculture. In: Genetic Resources of Phaseolus Beans. P Gepts (ed). Kluwer, Dordrecht, The Neetherlands. 619 p.

Gepts P (1999) What can molecular markers tell us about the process of domestication in common bean? In: The Origins of Agriculture and the Domestication of Crop Plants. A Damania, J Valkoun, G Willcox, C O Qualset (eds). International Center for Agricultural Research in the Dry Areas (ICARDA). Aleppo, Syria. pp:198-209.

Gepts P (2004) Crop domestication as a long-term selection experiment. In: Plant Breeding Reviews. J Janick (ed). Vol 24 Part 2. John Wiley \& Sons. New York. pp:1-44.

Gepts P, Bliss F A (1985) F1 hybrid weakness in the common bean: differential geographic origin suggests two gene pools in cultivated bean germplasm. J. Hered. 76:447-450.

Islam F M A, K E Basford, C Jara, R J Redden, S Beebe (2002a) Seed compositional and disease resistance differences among gene pools in cultivated common bean. Genet. Res. Crop Evol. 49:285-293.

Islam F M A, K E Basford, C Jara, R J Redden, A V Gonzalez, P M Kroonenberg, S Beebe (2002b) Genetic variability in cultivated bean beyond the two major gene pools. Genet. Res. Crop. Evol. 49:271-283.

Kaplan L (1965) Archeology and domestication in American Phaseolus (beans). Econ. Bot. 19:358-368.

Kaplan L, T F Lynch, C E Smith (1973) Early cultivated beans (Phaseolus vulgaris) from an intermontane Peruvian valley. Science 179:76-77.

Koenig R, P Gepts (1989) Allozyme diversity in wild Phaseolus vulgaris: further evidence for two major centers of genetic diversity. Theor. Appl. Genet. 78:809-817.

Kwak M, P Gepts (2009) Structure of genetic diversity in the two major gene pools of common bean (Phaseolus vulgaris L., Fabaceae). Theor. Appl. Genet. 118:979-992.

Kwak M, J A Kami, P Gepts (2009) The putative Mesoamerican domestication center of Phaseolus vulgaris is located in the LermaSantiago Basin of Mexico. Crop Sci. 49:554-563.

Ladizinsky G (1998) Plant evolution under domestication. Kluwer Academic Publishers. Dordrecht, The Netherlands. 256 p.

Lareo L R, A V González, E Barona, S D. Beebe (1993) Fast and reliable one and two-dimensional electrophoretic parameters for phaseolin type identification. Annu. Rep. Bean Improv. Coop. 36:8-9.

Mamidi S, M Rossi, D Annam, S Moghaddam, R Lee, R Papa, P McClean (2011) Investigation of the domestication of common bean (Phaseolus vulgaris) using multilocus sequence data. Funct. Plant Biol. 38:953-967.

Matsuoka Y, Y Vigouroux, M M Goodman, J Sanchez, E Buckler, J Doebley (2002) A single domestication for maize shown by multilocus microsatellite genotyping. Proc. Nat. Acad. Sci. USA 99:6080-6084.

Mensack M M, V K Fitzgerald, E P Ryan, M R Lewis, H J Thompson, M A Brick (2010) Evaluation of diversity among common beans
(Phaseolus vulgaris L.) from two centers of domestication using 'omics' technologies. BMC Genomics 11:686.

Mercado-Ruaro P, A Delgado-Salinas (2000) Cytogenetics studies in Phaseolus L. (Fabaceae). Genet. Mol. Biol. 23:985-987.

Miranda-Colín S (1967) Origen de Phaseolus vulgaris L. (frijol común). Agrociencia 1:99-104

Nanni L, E Bitocchi, E Bellucci, M Rossi, D Rau, G Attene, P Gepts, R Papa (2011) Nucleotide diversity of a genomic sequence similar to SHATTERPROOF (PvSHP1) in domesticated and wild common bean (Phaseolus vulgaris L.). Theor. Appl. Genet. 123: 1341-1357.

Papa R, Gepts P L. (2003) Asymmetry of gene flow and differential geographical structure of molecular diversity in wild and domesticated common bean (Phaseolus vulgaris L.) from Mesoamerica. Theor. Appl. Genet. 106:239-250.

Papa R, L Nanni, D Sicard, D Rau, G Attene (2006) The evolution of genetic diversity in Phaseolus vulgaris L. In: Darwin's Harvest: New Approaches to the Origins, Evolution, and Conservation of Crops. J J Motley, N Zerega, H Cross (eds). Columbia University Press. New York. pp:121-142.

Peña-Valdivia C B, J R García-Nava, J R Aguirre, M C Ybarra-Moncada, M López (2011) Variation in physical and chemical characteristics of common bean (Phaseolus vulgaris L.) grain along a domestication gradient. Chem. Biodiv. 8:2211-2225.

Pickersgill B (1989) Cytological and Genetical Evidence on the Domestication and Diffusion of Crops within the Americas. In: Foraging and Farming: The Evolution of Plant Exploitation. D R Harris, G C Hillman (eds). Unwin Hyman, London. pp:426-439.

Pope K O, M E D Pohl, J G Jones, D L Lentz, C V Nagy, F J Vega, I R Quitmyer (2001) Origin and environmental setting of ancient agriculture in the lowlands of Mesoamerica. Science 292:1370-1373.

Rossi M, E Bitocchi, E Bellucci, L Nanni, D Rau, G Attene, R Papa (2009) Linkage disequilibrium and population structure in wild and domesticated populations of Phaseolus vulgaris $\mathrm{L}$. Evol. Appl. 2:504-522.

Sanjur O I, D R Piperno, T C Andres, L Wessel-Beaver (2002) Phylogenetic relationships among domesticated and wild species of Cucurbita (Cucurbitaceae) inferred from a mitocondrial gene: implications for crop plant evolution and areas of origin. Proc. Natl. Acad. Sci. USA 99:535-540.

Schoonhoven A V, O Voysest (1991) Common Beans. Research for Crop Improvement. CAB International,Wallingford, UK, CIAT, Cali, Colombia. $980 \mathrm{p}$.

Singh S P, J A Gutierrez, A Molina, C Urrea, P Gepts (1991a) Genetic diversity in cultivated common bean: II. Marker-based analysis of morphological and agronomic traits. Crop Sci. 31:23-29.

Singh S P, P Gepts, D G Debouck (1991b) Races of common bean (Phaseolus vulgaris, Fabaceae). Econ. Bot. 45:379-396.

Smith B D (1997) The initial domestication of Cucurbita pepo in the Americas 10,000 years ago. Science 276:932-934.

Smith B D (2001) Documenting plant domestication: The consilience of biological and archaeological approaches. Proc. Natl. Acad. Sci. USA 94:1324-1326.

Smith B D (2005) Reassessing Coxcatlan Cave and the early history of domesticated plants in Mesoamerica. Proc. Natl. Acad. Sci. USA 102:9438-9445.

Smith B D (2006) Eastern North America as an independent center of plant domestication. Proc. Natl. Acad. Sci. USA 103:1222312228

Sonnante G, T Stockton, R O Nodari, V L Becerra-Velásquez, P Gepts (1994) Evolution of genetic diversity during the domestication of common-bean (Phaseolus vulgaris L.). Theor. Appl. Genet. 89:629-635.

Vargas M L P, J S Muruaga, J A Acosta, R Navarrete, P Pérez, G Esquivel, M B G Irízar, J M Hernández (2006) Colección Núcleo de Phaseolus vulgaris L. del INIFAP. Catálogo de Accesiones de la Forma Domesticada. Libro Técnico no. 10. Instituto Nacional de Investigaciones Forestales, Agrícolas y Pecuarias, Campo Experimental Valle de México. Chapingo, México. 461 p.

Zizumbo-Villarreal D, P Colunga-GarcíaMarín (2010) Origin of agriculture and plant domestication in West Mesoamerica. Genet. Res. Crop Evol. 57:813-825.

Zohary D (1999) Monophyletic vs. polyphyletic origin of the crops on which agriculture was founded in the Near East. Genet. Res. Crop Evol. 46:133-142. 